\title{
Asymptotic convergence of constrained primal-dual dynamics
}

\author{
Ashish Cherukuri ${ }^{\mathrm{a}}$, Enrique Mallada ${ }^{\mathrm{b}}$, Jorge Cortés $^{\mathrm{a}}$ \\ ${ }^{a}$ Department of Mechanical and Aerospace Engineering, University of California, San \\ Diego, CA 92093, USA \\ ${ }^{b}$ Department of Computational and Mathematical Sciences, California Institute of \\ Technology, Pasadena, CA 91125, USA
}

\begin{abstract}
This paper studies the asymptotic convergence properties of the primal-dual dynamics designed for solving constrained concave optimization problems using classical notions from stability analysis. We motivate the need for this study by providing an example that rules out the possibility of employing the invariance principle for hybrid automata to study asymptotic convergence. We understand the solutions of the primal-dual dynamics in the Caratheodory sense and characterize their existence, uniqueness, and continuity with respect to the initial condition. We use the invariance principle for discontinuous Caratheodory systems to establish that the primal-dual optimizers are globally asymptotically stable under the primal-dual dynamics and that each solution of the dynamics converges to an optimizer.
\end{abstract}

Keywords: primal-dual dynamics; constrained optimization; saddle points; discontinuous dynamics; Caratheodory solutions

\section{Introduction}

The (constrained) primal-dual dynamics is a widespread continuous-time algorithm for determining the primal and dual solutions of an inequality constrained convex (or concave) optimization problem. This dynamics, first

Email addresses: acheruku@ucsd.edu (Ashish Cherukuri), mallada@caltech.edu (Enrique Mallada), cortes@ucsd.edu (Jorge Cortés) 
introduced in the pioneering works $[1,2]$, has been used in multiple applications, including network resource allocation problems for wireless systems $[3,4,5]$ and distributed stabilization and optimization of power networks $[6,7,8,9]$.

Our objective in this paper is to provide a rigorous treatment of the convergence analysis of the primal-dual dynamics using classical notions from stability analysis. Since this dynamics has a discontinuous right-hand side, the standard Lyapunov or LaSalle-based stability results for nonlinear systems, see e.g. [10], are not directly applicable. This observation is at the basis of the direct approach to establish convergence taken in [1], where the evolution of the distance of the solution of the primal-dual dynamics to an arbitrary primal-dual optimizer is approximated using power series expansions and its monotonic evolution is concluded by analyzing the local behavior around a saddle point of the terms in the series. Instead, [3] takes an indirect approach to establish convergence, modeling the primal-dual dynamics as a hybrid automaton as defined in [11], and invoking a generalized LaSalle Invariance Principle to establish asymptotic convergence. However, the hybrid automaton that corresponds to the primal-dual dynamics is in general not continuous, thereby not satisfying a key requirement of the invariance principle stated in [11], and invalidating this route to establish convergence. The first contribution of this paper is an example that illustrates this point. Our second contribution is an alternative proof strategy to arrive at the same convergence results of [3].

For the problem setup, we consider an inequality constrained concave optimization problem described by continuously differentiable functions with locally Lipschitz gradients. Since the primal-dual dynamics has a discontinuous right-hand side, we specify the notion of solution in the Caratheodory sense (note that this does not necessarily preclude the study of other notions of solution). We show that the primal-dual dynamics is a particular case of a projected dynamical system and, using results from [12], we establish that Caratheodory solutions exist, are unique, and are continuous with respect to the initial condition. Using these properties, we show that the omega-limit set of any solution of the primal-dual dynamics is invariant under the dynamics. Finally, we employ the invariance principle for Caratheodory solutions of discontinuous dynamical systems from [13] to show that the primal-dual optimizers are globally asymptotically stable under the primal-dual dynamics and that each solution of the dynamics converges to an optimizer. We 
believe the use of classical notions of stability and Lyapunov methods provides a conceptually simple and versatile approach that can also be invoked in characterizing other properties of the dynamics.

The paper is organized as follows. Section 2 presents basic notation and preliminary notions on discontinuous dynamical systems. Section 3 introduces the primal-dual dynamics and motivates with an example the need for a convergence analysis with classical stability tools. Section 4 presents the main convergence results. Finally, Section 5 gathers our conclusions and ideas for future work.

\section{Preliminaries}

This section introduces notation and basic concepts about discontinuous and projected dynamical systems.

\subsection{Notation}

We let $\mathbb{R}, \mathbb{R}_{\geq 0}, \mathbb{R}_{>0}$, and $\mathbb{Z}_{\geq 1}$ be the set of real, nonnegative real, positive real, and positive integer numbers, respectively. We denote by $\|\cdot\|$ the 2 norm on $\mathbb{R}^{n}$. The open ball of radius $\delta>0$ centered at $x \in \mathbb{R}^{n}$ is represented by $B_{\delta}(x)$. Given $x \in \mathbb{R}^{n}, x_{i}$ denotes the $i$-th component of $x$. For $x, y \in \mathbb{R}^{n}$, $x \leq y$ if and only if $x_{i} \leq y_{i}$ for all $i \in\{1, \ldots, n\}$. We use the shorthand notation $\mathbf{0}_{n}=(0, \ldots, 0) \in \mathbb{R}^{n}$. For a real-valued function $V: \mathbb{R}^{n} \rightarrow \mathbb{R}$ and $\alpha>0$, we denote the sublevel set of $V$ by $V^{-1}(\leq \alpha)=\left\{x \in \mathbb{R}^{n} \mid V(x) \leq \alpha\right\}$. For scalars $a, b \in \mathbb{R}$, the operator $[a]_{b}^{+}$is defined as

$$
[a]_{b}^{+}= \begin{cases}a, & \text { if } b>0 \\ \max \{0, a\}, & \text { if } b=0\end{cases}
$$

For vectors $a, b \in \mathbb{R}^{n},[a]_{b}^{+}$denotes the vector whose $i$-th component is $\left[a_{i}\right]_{b_{i}}^{+}$, $i \in\{1, \ldots, n\}$. For a set $\mathcal{S} \in \mathbb{R}^{n}$, its interior, closure, and boundary are denoted by $\operatorname{int}(\mathcal{S}), \operatorname{cl}(\mathcal{S})$, and $\operatorname{bd}(\mathcal{S})$, respectively. Given two sets $X$ and $Y$, a set-valued map $f: X \rightrightarrows Y$ associates to each point in $X$ a subset of $Y$. A map $f: \mathbb{R}^{n} \rightarrow \mathbb{R}^{m}$ is locally Lipschitz at $x \in \mathbb{R}^{n}$ if there exist $\delta_{x}, L_{x}>0$ such that $\left\|f\left(y_{1}\right)-f\left(y_{2}\right)\right\| \leq L_{x}\left\|y_{1}-y_{2}\right\|$ for any $y_{1}, y_{2} \in B_{\delta_{x}}(x)$. If $f$ is locally Lipschitz at every $x \in \mathcal{K} \subset \mathbb{R}^{n}$, then we simply say that $f$ is locally Lipschitz on $\mathcal{K}$. The map $f$ is Lipschitz on $\mathcal{K} \subset \mathbb{R}^{n}$ if there exists a constant $L>0$ 
such that $\|f(x)-f(y)\| \leq L\|x-y\|$ for any $x, y \in \mathcal{K}$. Note that if $f$ is locally Lipschitz on $\mathbb{R}^{n}$, then it is Lipschitz on every compact set $\mathcal{K} \subset \mathbb{R}^{n}$. The map $f$ is locally bounded if for each $x \in \mathbb{R}^{n}$ there exists constants $M_{x}, \epsilon_{x}>0$ such that $\|f(y)\| \leq M_{x}$ for all $y \in B_{\epsilon_{x}}(x)$.

\subsection{Discontinuous dynamical systems}

Here we present basic concepts on discontinuous dynamical systems following $[13,14]$. Let $f: \mathbb{R}^{n} \rightarrow \mathbb{R}^{n}$ be Lebesgue measurable and locally bounded and consider the differential equation

$$
\dot{x}=f(x) .
$$

A map $\gamma:[0, T) \rightarrow \mathbb{R}^{n}$ is a (Caratheodory) solution of (1) on the interval $[0, T)$ if it is absolutely continuous on $[0, T)$ and satisfies $\dot{\gamma}(t)=f(\gamma(t))$ almost everywhere in $[0, T)$. A set $\mathcal{S} \subset \mathbb{R}^{n}$ is invariant under (1) if every solution starting from any point in $\mathcal{S}$ remains in $\mathcal{S}$. For a solution $\gamma$ of (1) defined on the time interval $[0, \infty)$, the omega-limit set $\Omega(\gamma)$ is defined by

$$
\Omega(\gamma)=\left\{y \in \mathbb{R}^{n} \mid \exists\left\{t_{k}\right\}_{k=1}^{\infty} \subset[0, \infty) \text { with } \lim _{k \rightarrow \infty} t_{k}=\infty \text { and } \lim _{k \rightarrow \infty} \gamma\left(t_{k}\right)=y\right\}
$$

If the solution $\gamma$ is bounded, then $\Omega(\gamma) \neq \emptyset$ by the Bolzano-Weierstrass theorem [15]. These notions allow us to characterize the asymptotic convergence properties of the solutions of (1) via invariance principles. Given a continuously differentiable function $V: \mathbb{R}^{n} \rightarrow \mathbb{R}$, the Lie derivative of $V$ along (1) at $x \in \mathbb{R}^{n}$ is $\mathcal{L}_{f} V(x)=\nabla V(x)^{\top} f(x)$. The next result is a simplified version of [13, Proposition 3] which is sufficient for our convergence analysis later.

Proposition 2.1. (Invariance principle for discontinuous Caratheodory systems): Let $\mathcal{S} \in \mathbb{R}^{n}$ be compact and invariant. Assume that, for each point $x_{0} \in \mathcal{S}$, there exists a unique solution of (1) starting at $x_{0}$ and that its omegalimit set is invariant too. Let $V: \mathbb{R}^{n} \rightarrow \mathbb{R}$ be a continuously differentiable map such that $\mathcal{L}_{f} V(x) \leq 0$ for all $x \in \mathcal{S}$. Then, any solution of (1) starting at $\mathcal{S}$ converges to the largest invariant set in $\operatorname{cl}\left(\left\{x \in \mathcal{S} \mid \mathcal{L}_{f} V(x)=0\right\}\right)$.

\subsection{Projected dynamical systems}

Projected dynamical systems are a particular class of discontinuous dynamical systems. Here, following [12], we gather some basic notions that 
will be useful later to establish continuity with respect to the initial condition of the solutions of the primal-dual dynamics. Let $\mathcal{K} \subset \mathbb{R}^{n}$ be a closed convex set. Given a point $y \in \mathbb{R}^{n}$, the (point) projection of $y$ onto $\mathcal{K}$ is $\operatorname{proj}_{\mathcal{K}}(y)=\operatorname{argmin}_{z \in \mathcal{K}}\|z-y\|$. Note that $\operatorname{proj}_{\mathcal{K}}(y)$ is a singleton and the map $\operatorname{proj}_{\mathcal{K}}$ is Lipschitz on $\mathbb{R}^{n}$ with constant $L=1$ [16, Proposition 2.4.1]. Given $x \in \mathcal{K}$ and $v \in \mathbb{R}^{n}$, the (vector) projection of $v$ at $x$ with respect to $\mathcal{K}$ is

$$
\Pi_{\mathcal{K}}(x, v)=\lim _{\delta \rightarrow 0^{+}} \frac{\operatorname{proj}_{\mathcal{K}}(x+\delta v)-x}{\delta} .
$$

Given a vector field $f: \mathbb{R}^{n} \rightarrow \mathbb{R}^{n}$ and a closed convex polyhedron $\mathcal{K} \subset \mathbb{R}^{n}$, the associated projected dynamical system is

$$
\dot{x}=\Pi_{\mathcal{K}}(x, f(x)), \quad x(0) \in \mathcal{K},
$$

Note that, at any point $x$ in the interior of $\mathcal{K}$, we have $\Pi_{\mathcal{K}}(x, f(x))=f(x)$. At any boundary point of $\mathcal{K}$, the projection operator restricts the flow of the vector field $f$ such that the solutions of $(2)$ remain in $\mathcal{K}$. Therefore, in general, (2) is a discontinuous dynamical system. The next result summarizes conditions under which the (Caratheodory) solutions of the projected system (2) exist, are unique, and continuous with respect to the initial condition.

Proposition 2.2. (Existence, uniqueness, and continuity with respect to the initial condition [12, Theorem 2.5]): Let $f: \mathbb{R}^{n} \rightarrow \mathbb{R}^{n}$ be Lipschitz on a closed convex polyhedron $\mathcal{K} \subset \mathbb{R}^{n}$. Then,

(i) (existence and uniqueness): for any $x_{0} \in \mathcal{K}$, there exists a unique solution $t \mapsto x(t)$ of the projected system (2) with $x(0)=x_{0}$ defined over the domain $[0, \infty)$,

(ii) (continuity with respect to the initial condition): given a sequence of points $\left\{x_{k}\right\}_{k=1}^{\infty} \subset \mathcal{K}$ with $\lim _{k \rightarrow \infty} x_{k}=x$, the sequence of solutions $\left\{t \mapsto \gamma_{k}(t)\right\}_{k=1}^{\infty}$ of $(2)$ with $\gamma_{k}(0)=x_{k}$ for all $k$, converge to the solution $t \mapsto \gamma(t)$ of $(2)$ with $\gamma(0)=x$ uniformly on every compact set of $[0, \infty)$.

\section{Problem statement}

This section reviews the primal-dual dynamics for solving constrained optimization problems and justifies the need to rigorously characterize its 
convergence properties. Consider the concave optimization problem on $\mathbb{R}^{n}$,

$$
\begin{aligned}
\text { maximize } & f(x), \\
\text { subject to } & g(x) \leq \mathbf{0}_{m},
\end{aligned}
$$

where the continuously differentiable functions $f: \mathbb{R}^{n} \rightarrow \mathbb{R}$ and $g: \mathbb{R}^{n} \rightarrow$ $\mathbb{R}^{m}$ are strictly concave and convex, respectively, and have locally Lipschitz gradients. The Lagrangian of the problem (3) is given as

$$
L(x, \lambda)=f(x)-\lambda^{\top} g(x),
$$

where $\lambda \in \mathbb{R}^{m}$ is the Lagrange multiplier corresponding to the inequality constraint (3b). Note that the Lagrangian is concave in $x$ and convex (in fact linear) in $\lambda$. Assume that the Slater's conditions is satisfied for the problem (3), that is, there exists $x \in \mathbb{R}^{n}$ such that $g(x)<\mathbf{0}_{m}$. Under this assumption, the duality gap between the primal and dual optimizers is zero and a point $\left(x_{*}, \lambda_{*}\right) \in \mathbb{R}^{n} \times \mathbb{R}_{\geq 0}^{m}$ is a primal-dual optimizer of (3) if and only if it is a saddle point of $L$ over the domain $\mathbb{R}^{n} \times \mathbb{R}_{\geq 0}^{m}$, i.e.,

$$
L(x, \lambda) \leq L\left(x_{*}, \lambda_{*}\right) \quad \text { and } \quad L\left(x_{*}, \lambda\right) \geq L\left(x_{*}, \lambda_{*}\right),
$$

for all $x \in \mathbb{R}^{n}$ and $\lambda \in \mathbb{R}_{\geq 0}^{m}$. For convenience, we denote the set of saddle points of $L$ (equivalently the primal-dual optimizers) by $\mathrm{X} \times \Lambda \subset \mathbb{R}^{n} \times \mathbb{R}^{m}$. Note that since $f$ is strictly concave, the set $\mathbf{X}$ is a singleton. Furthermore, $\left(x_{*}, \lambda_{*}\right)$ is a primal-dual optimizer if and only if it satisfies the following Karush-Kuhn-Tucker (KKT) conditions (cf. [17, Chapter 5]),

$$
\begin{array}{r}
\nabla f\left(x_{*}\right)-\sum_{i=1}^{m}\left(\lambda_{*}\right)_{i} \nabla g_{i}\left(x_{*}\right)=0, \\
g\left(x_{*}\right) \leq \mathbf{0}_{m}, \quad \lambda_{*} \geq \mathbf{0}_{m}, \quad \lambda_{*}^{\top} g\left(x_{*}\right)=0 .
\end{array}
$$

Given this characterization of the solutions of the optimization problem, it is natural to consider the primal-dual dynamics on $\mathbb{R}^{n} \times \mathbb{R}_{\geq 0}^{m}$ to find them

$$
\begin{aligned}
& \dot{x}=\nabla_{x} L(x, \lambda)=\nabla f(x)-\sum_{i=1}^{m} \lambda_{i} \nabla g_{i}(x), \\
& \dot{\lambda}=\left[-\nabla_{\lambda} L(x, \lambda)\right]_{\lambda}^{+}=[g(x)]_{\lambda}^{+} .
\end{aligned}
$$


When convenient, we use the notation $X_{\mathrm{p}-\mathrm{d}}: \mathbb{R}^{n} \times \mathbb{R}_{\geq 0}^{m} \rightarrow \mathbb{R}^{n} \times \mathbb{R}^{m}$ to refer to the dynamics (6). Given that the primal-dual dynamics is discontinuous, we consider solutions in the Caratheodory sense. The reason for this is that, with this notion of solution, a point is an equilibrium of (6) if and only if it satisfies the KKT conditions (5).

Our objective is to establish that the solutions of (6) exist and asymptotically converge to a solution of the concave optimization problem (3) using classical notions and tools from stability analysis. Our motivation for this aim comes from the conceptual simplicity and versatility of Lyapunovlike methods and their amenability for performing robustness analysis and studying generalizations of the dynamics. One way of tackling this problem, see e.g., [3], is to interpret the dynamics as a state-dependent switched system, formulate the latter as a hybrid automaton as defined in [11], and then employ the invariance principle for hybrid automata to characterize its asymptotic convergence properties. However, this route is not valid in general because one of the key assumptions required by the invariance principle for hybrid automata is not satisfied by the primal-dual dynamics. The next example justifies this claim.

Example 3.1. (The hybrid automaton corresponding to the primal-dual dynamics is not continuous): Consider the concave optimization problem (3) on $\mathbb{R}$ with $f(x)=-(x-5)^{2}$ and $g(x)=x^{2}-1$, whose set of primal-dual optimizers is $\mathrm{X} \times \Lambda=\{(1,4)\}$. The associated primal-dual dynamics takes the form

$$
\begin{aligned}
& \dot{x}=-2(x-5)-2 x \lambda, \\
& \dot{\lambda}=\left[x^{2}-1\right]_{\lambda}^{+} .
\end{aligned}
$$

We next formulate this dynamics as a hybrid automaton as defined in $[11$, Definition II.1]. The idea to build the hybrid automaton is to divide the state space $\mathbb{R} \times \mathbb{R}_{\geq 0}$ into two domains over which the vector field (7) is continuous. To this end, we define two modes represented by the discrete variable $q$, taking values in $\mathbf{Q}=\{1,2\}$. The value $q=1$ represents the mode where the projection in $(7 \mathrm{~b})$ is active and $q=2$ represents the mode where it is not. Formally, the projection is active at $(x, \lambda)$ if $[g(x)]_{\lambda}^{+} \neq g(x)$, i.e, $\lambda=0$ and $g(x)<0$. The hybrid automaton is then given by the collection $H=(Q, X, f$, Init, $D, E, G, R)$, where $Q=\{q\}$ is the set of discrete variables, 
taking values in $\mathbf{Q} ; X=\{x, \lambda\}$ is the set of continuous variables, taking values in $\mathbf{X}=\mathbb{R} \times \mathbb{R}_{\geq 0}$; the vector field $f: \mathbf{Q} \times \mathbf{X} \rightarrow T \mathbf{X}$ is defined by

$$
\begin{aligned}
& f(1,(x, \lambda))=\left[\begin{array}{c}
-2(x-5)-2 x \lambda \\
0
\end{array}\right], \\
& f(2,(x, \lambda))=\left[\begin{array}{c}
-2(x-5)-2 x \lambda \\
x^{2}-1
\end{array}\right] ;
\end{aligned}
$$

Init $=\mathbf{X}$ is the set of initial conditions; $D: \mathbf{Q} \rightrightarrows \mathbf{X}$ specifies the domain of each discrete mode,

$$
D(1)=(-1,1) \times\{0\}, \quad D(2)=\mathbf{X} \backslash D(1),
$$

i.e., the dynamics is defined by the vector field $(x, \lambda) \rightarrow f(1,(x, \lambda))$ over $D(1)$ and by $(x, \lambda) \rightarrow f(2,(x, \lambda))$ over $D(2) ; E=\{(1,2),(2,1)\}$ is the set of edges specifying the transitions between modes; the guard map $G: \mathbf{Q} \rightrightarrows \mathbf{X}$ specifies when a solution can jump from one mode to the other,

$$
G(1,2)=\{(1,0),(-1,0)\}, \quad G(2,1)=(-1,1) \times\{0\},
$$

i.e., $G\left(q, q^{\prime}\right)$ is the set of points where a solution jumps from mode $q$ to mode $q^{\prime}$; and, finally, the reset map $R: \mathbf{Q} \times \mathbf{X} \rightrightarrows \mathbf{X}$ specifies that the state is preserved after a jump from one mode to another,

$$
R((1,2),(x, \lambda))=R((2,1),(x, \lambda))=\{(x, \lambda)\} .
$$

We are now ready to show that the hybrid automaton is not continuous in the sense defined by [11, Definition III.3]. This notion plays a key role in the study of omega-limit sets and their stability, and is in fact a basic assumption of the invariance principle developed in [11, Theorem IV.1]. Roughly speaking, $H$ is continuous if two executions of $H$ starting close to one another remain close to one another. An execution of $H$ consists of a tuple $(\tau, q, x)$, where $\tau$ is a hybrid time trajectory (a sequence of intervals specifying where mode transitions and continuous evolution take place), $q$ is a map that gives the discrete mode of the execution at each interval of $\tau$, and $x$ is a set of differentiable maps that represent the evolution of the continuous state of the execution along intervals of $\tau$. A necessary condition for two executions to "remain close" is to have the time instants of transitions in their mode for the executions (if there are any) close to one another. 


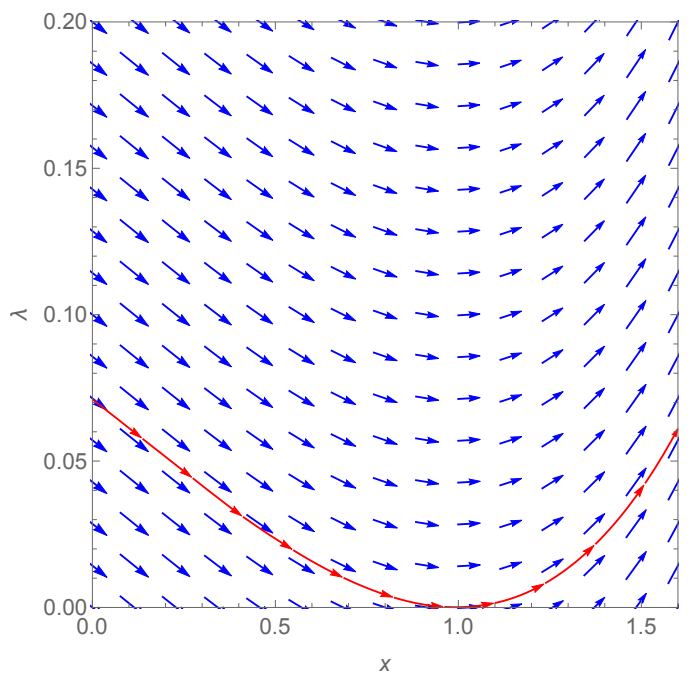

Figure 1: An illustration depicting the vector field $(7)$ in the range $(x, \lambda) \in[0,1.6] \times[0,0.2]$. As shown (with a red streamline), there exists a solution of (7) that starts at a point $(x(0), \lambda(0))$ with $x(0)<1$ and $\lambda(0)>0$ such that it remains in the domain $\lambda>0$ at all times except at one time instant $t^{\prime}$ when $\left(x\left(t^{\prime}\right), \lambda\left(t^{\prime}\right)\right)=(1,0)$.

To disprove the continuity of $H$, it is enough then to show that there exist two executions that start arbitrarily close and yet experience their first mode transitions at time instants that are not arbitrarily close. Select an initial condition $(x(0), \lambda(0)) \in(0,1) \times(0, \infty)$ that gives rise to a solution of $(7)$ that remains in the set $(0,1) \times(0, \infty)$ for a finite time interval $\left(0, t^{\prime}\right)$, $t^{\prime}>0$, satisfies $\left(x\left(t^{\prime}\right), \lambda\left(t^{\prime}\right)\right)=(1,0)$, and stays in the set $(1, \infty) \times(0, \infty)$ for some finite time interval $\left(t^{\prime}, T\right), T>t^{\prime}$. The existence of such a solution becomes clear by plotting the vector field (7), see Figure 1 . Note that by construction, this also corresponds to an execution of the hybrid automaton $H$ that starts and remains in domain $D(2)$ for the time interval $[0, T]$ and so it does not encounter any jumps in its discrete mode. Specifically, for this execution, the hybrid time trajectory is the interval $[0, T]$, the discrete mode $q$ is always 2 and the continuous state evolves as $t \mapsto(x(t), \lambda(t))$. Further, by observing the vector field, we deduce that in every neighborhood of $(x(0), \lambda(0))$, there exists a point $(\tilde{x}(0), \tilde{\lambda}(0))$ such that a solution of $(7)$ $t \mapsto(\tilde{x}(t), \tilde{\lambda}(t))$ starting at $(\tilde{x}(0), \tilde{\lambda}(0))$ reaches the set $(0,1) \times\{0\}$ in finite time $t_{1}>0$, remains in $(0,1) \times\{0\}$ for a finite time interval $\left[t_{1}, t_{2}\right]$, and then enters the set $(1, \infty) \times(0, \infty)$ upon reaching the point $(1,0)$. Indeed, this is true whenever $\tilde{x}<x(0)$ and $\tilde{\lambda}<\lambda(0)$. The execution of $H$ corresponding 
to this solution starts in $D(2)$, enters $D(1)$ in finite time $t_{1}$, and returns to $D(2)$ at time $t_{2}$. Specifically, the hybrid time trajectory consists of three intervals $\left\{\left[0, t_{1}\right],\left[t_{1}, t_{2}\right],\left[t_{2}, T^{\prime}\right]\right\}$, where we assume $T^{\prime}>t_{2}$. The discrete mode $q$ takes value 2 for the interval $\left[0, t_{1}\right], 1$ for the interval $\left[t_{1}, t_{2}\right]$, and 2 for the interval $\left[t_{2}, T^{\prime}\right]$. The continuous state $t \mapsto(\tilde{x}(t), \tilde{\lambda}(t))$ takes the same values as the solution of (7) explained above. Thus, the value of the discrete variable representing the mode of the execution switches from 2 to 1 and back to 2, whereas the execution corresponding to the solution of (7) starting at $(x(0), \lambda(0))$ never switches mode. This shows that the hybrid automaton is not continuous.

Interestingly, even though the hybrid automaton $H$ described in Example 3.1 is not continuous, one can infer from Figure 1 that two solutions of (7) remain close to each other if they start close enough. This suggests that continuity with respect to the initial condition might hold provided this notion is formalized the way it is done for traditional nonlinear systems (and not as done for hybrid automata where both discrete and continuous states have to be aligned). The next section shows that this in fact is the case. This, along with the existence and uniqueness of solutions, allows us to characterize the asymptotic convergence properties of the primal-dual dynamics.

\section{Convergence analysis of primal-dual dynamics}

In this section we show that the solutions of the primal-dual dynamics (6) asymptotically converge to a solution of the constrained optimization problem (3). Our proof strategy is to employ the invariance principle for Caratheodory solutions of discontinuous dynamical systems stated in Proposition 2.1. Our first step is then to verify that all its hypotheses hold.

We start by stating a useful monotonicity property of the primal-dual dynamics with respect to the set of primal-dual optimizers $X \times \Lambda$. This property can be found in $[1,3]$ and we include here its proof for completeness.

Lemma 4.1. (Monotonicity of the primal-dual dynamics with respect to primal-dual optimizers): Let $\left(x_{*}, \lambda_{*}\right) \in \mathbf{X} \times \Lambda$ and define $V: \mathbb{R}^{n} \times \mathbb{R}^{m} \rightarrow \mathbb{R}_{\geq 0}$,

$$
V(x, \lambda)=\frac{1}{2}\left(\left\|x-x_{*}\right\|^{2}+\left\|\lambda-\lambda_{*}\right\|^{2}\right) .
$$


Then $\mathcal{L}_{X_{p-d}} V(x, \lambda) \leq 0$ for all $(x, \lambda) \in \mathbb{R}^{n} \times \mathbb{R}_{\geq 0}^{m}$.

Proof. By definition of $\mathcal{L}_{X_{\mathrm{p}-\mathrm{d}}} V$ (cf. Section 2.2), we have

$$
\begin{aligned}
\mathcal{L}_{X_{\mathrm{p}-\mathrm{d}}} V(x, \lambda)= & \left(x-x_{*}\right)^{\top} \nabla_{x} L(x, \lambda)+\left(\lambda-\lambda_{*}\right)^{\top}\left[-\nabla_{\lambda} L(x, \lambda)\right]_{\lambda}^{+} \\
= & \left(x-x_{*}\right)^{\top} \nabla_{x} L(x, \lambda)-\left(\lambda-\lambda_{*}\right)^{\top} \nabla_{\lambda} L(x, \lambda) \\
& +\left(\lambda-\lambda_{*}\right)^{\top}\left(\left[-\nabla_{\lambda} L(x, \lambda)\right]_{\lambda}^{+}+\nabla_{\lambda} L(x, \lambda)\right) .
\end{aligned}
$$

Since $L$ is concave in $x$ and convex in $\lambda$, applying the first order condition of concavity and convexity for the first two terms of the above expression yields the following bound

$$
\begin{aligned}
\mathcal{L}_{X_{\mathrm{p}-\mathrm{d}}} V(x, \lambda) \leq & L(x, \lambda)-L\left(x_{*}, \lambda\right)+L\left(x, \lambda_{*}\right)-L(x, \lambda) \\
& +\left(\lambda-\lambda_{*}\right)^{\top}\left(\left[-\nabla_{\lambda} L(x, \lambda)\right]_{\lambda}^{+}+\nabla_{\lambda} L(x, \lambda)\right) \\
= & L\left(x_{*}, \lambda_{*}\right)-L\left(x_{*}, \lambda\right)+L\left(x, \lambda_{*}\right)-L\left(x_{*}, \lambda_{*}\right) \\
& +\left(\lambda-\lambda_{*}\right)^{\top}\left(\left[-\nabla_{\lambda} L(x, \lambda)\right]_{\lambda}^{+}+\nabla_{\lambda} L(x, \lambda)\right) .
\end{aligned}
$$

Define the shorthand notation $M_{1}=L\left(x_{*}, \lambda_{*}\right)-L\left(x_{*}, \lambda\right), M_{2}=L\left(x, \lambda_{*}\right)-$ $L\left(x_{*}, \lambda_{*}\right)$, and $M_{3}=\left(\lambda-\lambda_{*}\right)^{\top}\left(\left[-\nabla_{\lambda} L(x, \lambda)\right]_{\lambda}^{+}+\nabla_{\lambda} L(x, \lambda)\right)$, so that the above inequality reads

$$
\mathcal{L}_{X_{\mathrm{p}-\mathrm{d}}} V(x, \lambda) \leq M_{1}+M_{2}+M_{3}
$$

Since $\lambda_{*}$ is a minimizer of the map $\lambda \rightarrow L\left(x_{*}, \lambda\right)$ over the domain $\mathbb{R}_{\geq 0}^{m}$ and $x_{*}$ is a maximizer of the map $x \rightarrow L\left(x, \lambda_{*}\right)$, we obtain $M_{1}, M_{2} \leq 0$. Replacing $-\nabla_{\lambda} L(x, \lambda)=g(x)$, one can write $M_{3}=\sum_{i=1}^{m} T_{i}$, where for each $i$,

$$
T_{i}=\left(\lambda_{i}-\left(\lambda_{*}\right)_{i}\right)\left(\left[g_{i}(x)\right]_{\lambda_{i}}^{+}-g_{i}(x)\right) .
$$

If $\lambda_{i}>0$, then $\left[g_{i}(x)\right]_{\lambda_{i}}^{+}=g_{i}(x)$ and so $T_{i}=0$. If $\lambda_{i}=0$, then $\lambda_{i}-\left(\lambda_{*}\right)_{i} \leq 0$ and $\left[g_{i}(x)\right]_{\lambda_{i}}^{+}-g_{i}(x) \geq 0$, which implies that $T_{i} \leq 0$. Therefore, we get $M_{3} \leq 0$, and the result follows.

Next, we show that the primal-dual dynamics can be written as a projected dynamical system.

Lemma 4.2. (Primal-dual dynamics as a projected dynamical system): The primal-dual dynamics can be written as a projected dynamical system. 
Proof. Consider the vector field $X: \mathbb{R}^{n} \times \mathbb{R}^{m} \rightarrow \mathbb{R}^{n} \times \mathbb{R}^{m}$ defined by

$$
X(x, \lambda)=\left[\begin{array}{c}
\nabla_{x} L(x, \lambda) \\
-\nabla_{\lambda} L(x, \lambda)
\end{array}\right] .
$$

We wish to show that $X_{\mathrm{p}-\mathrm{d}}(x, \lambda)=\prod_{\mathbb{R}^{n} \times \mathbb{R}_{\geq 0}^{m}}((x, \lambda), X(x, \lambda))$ for all $(x, \lambda) \in$ $\mathbb{R}^{n} \times \mathbb{R}_{\geq 0}^{m}$. To see this, note that the maps $X_{\mathrm{p}-\mathrm{d}}$ and $X$ take the same values over $\operatorname{int}\left(\mathbb{R}^{n} \times \mathbb{R}_{>0}^{m}\right)=\mathbb{R}^{n} \times \mathbb{R}_{>0}^{m}$. Now consider any point $(x, \lambda) \in$ $\operatorname{bd}\left(\mathbb{R}^{n} \times \mathbb{R}_{>0}^{m}\right)$. Let $I \subset\{1, \ldots, m\}$ be the set of indices for which $\lambda_{i}=0$ and $\left(-\nabla_{\lambda} L(x, \bar{\lambda})\right)_{i}<0$. Then, there exist $\tilde{\delta}>0$ such that, for all $\delta \in[0, \tilde{\delta})$ and for any $j \in\{1, \ldots, n+m\}$, we have

$$
\left(\operatorname{proj}_{\mathbb{R}^{n} \times \mathbb{R}_{\geq 0}^{m}}((x, \lambda)+\delta X(x, \lambda))\right)_{j}= \begin{cases}0, & \text { if } j-n \in I, \\ (x, \lambda)_{j}+\delta(X(x, \lambda))_{j}, & \text { otherwise } .\end{cases}
$$

Consequently, using the definition of the projection operator, cf. Section 2.3, we get

$$
\left(\Pi_{\mathbb{R}^{n} \times \mathbb{R}_{\geq 0}^{m}}((x, \lambda), X(x, \lambda))\right)_{j}= \begin{cases}0, & \text { if } j-n \in I, \\ (X(x, \lambda))_{j}, & \text { otherwise }\end{cases}
$$

which implies $X_{\mathrm{p}-\mathrm{d}}(x, \lambda)=\Pi_{\mathbb{R}^{n} \times \mathbb{R}_{\geq 0}^{m}}((x, \lambda), X(x, \lambda))$ for all $(x, \lambda) \in \mathrm{bd}\left(\mathbb{R}^{n} \times\right.$ $\mathbb{R}_{\geq 0}^{m}$ ). This concludes the proof.

Next, we use Lemmas 4.1 and 4.2 to show the existence, uniqueness, and continuity of the solutions of $X_{\mathrm{p}-\mathrm{d}}$ starting from $\mathbb{R}^{n} \times \mathbb{R}_{\geq 0}^{m}$. Our proof strategy consists of using Lemma 4.2 and Proposition 2.2 to conclude the result. A minor technical hurdle in this process is ensuring the Lipschitz property of the vector field (9), the projection of which on $\mathbb{R}^{n} \times \mathbb{R}_{>0}^{m}$ is $X_{\mathrm{p}-\mathrm{d}}$. We tackle this by using the monotonicity property of the primal-dual dynamics stated in Lemma 4.1 implying that a solution of $X_{\mathrm{p}-\mathrm{d}}$ (if it exists) remains in a bounded set, which we know explicitly. This further implies that, given a starting point, there exists a bounded set such that the values of the vector field outside this set do not affect the solution starting at that point and hence, the vector field can be modified at the outside points without loss of generality to obtain the Lipschitz property. We make this construction explicit in the proof. 
Lemma 4.3. (Existence, uniqueness, and continuity of solutions of the primaldual dynamics): Starting from any point $(x, \lambda) \in \mathbb{R}^{n} \times \mathbb{R}_{\geq 0}^{m}$, a unique solution $t \mapsto \gamma(t)$ of the primal-dual dynamics $X_{p-d}$ exists and remains in $\left(\mathbb{R}^{n} \times \mathbb{R}_{\geq 0}^{m}\right) \cap$ $V^{-1}(\leq V(x, \lambda))$. Moreover, if a sequence of points $\left\{\left(x_{k}, \lambda_{k}\right)\right\}_{k=1}^{\infty} \subset \mathbb{R}^{n} \times \mathbb{R}_{\geq 0}^{m}$ converge to $(x, \lambda)$ as $k \rightarrow \infty$, then the sequence of solutions $\left\{t \mapsto \gamma_{k}(t)\right\}_{k=1}^{\infty}$ of $X_{p-d}$ starting at these points converge uniformly to the solution $t \mapsto \gamma(t)$ on every compact set of $[0, \infty)$.

Proof. Consider $(x(0), \lambda(0)) \in \mathbb{R}^{n} \times \mathbb{R}_{>0}^{m}$ and let $\epsilon>0$. Define $V_{0}=$ $V(x(0), \lambda(0))$, where $V$ is given in (8), and let $\mathcal{W}_{\epsilon}=V^{-1}\left(\leq V_{0}+\epsilon\right)$. Note that $\mathcal{W}_{\epsilon}$ is convex, compact, and $V^{-1}\left(\leq V_{0}\right) \subset \operatorname{int}\left(\mathcal{W}_{\epsilon}\right)$. Let $X^{\mathcal{W}_{\epsilon}}: \mathbb{R}^{n} \times \mathbb{R}^{m} \rightarrow$ $\mathbb{R}^{n} \times \mathbb{R}^{m}$ be a vector field defined as follows: equal to $X$ on $\mathcal{W}_{\epsilon}$ and, for any $(x, \lambda) \in\left(\mathbb{R}^{n} \times \mathbb{R}^{m}\right) \backslash \mathcal{W}_{\epsilon}$,

$$
X^{\mathcal{W}_{\epsilon}}(x, \lambda)=X\left(\operatorname{proj}_{\mathcal{W}_{\epsilon}}(x, \lambda)\right) .
$$

The vector field $X^{\mathcal{W}_{\epsilon}}$ is Lipschitz on the domain $\mathbb{R}^{n} \times \mathbb{R}^{m}$. To see this, note that $X$ is Lipschitz on the compact set $\mathcal{W}_{\epsilon}$ with some Lipschitz constant $K>$ 0 because $f$ and $g$ have locally Lipschitz gradients. Let $\left(x_{1}, \lambda_{1}\right),\left(x_{2}, \lambda_{2}\right) \in$ $\mathbb{R}^{n} \times \mathbb{R}^{m}$. Then,

$$
\begin{aligned}
\left\|X^{\mathcal{W}_{\epsilon}}\left(x_{1}, \lambda_{1}\right)-X^{\mathcal{W}_{\epsilon}}\left(x_{2}, \lambda_{2}\right)\right\| & =\left\|X\left(\operatorname{proj}_{\mathcal{W}_{\epsilon}}\left(x_{1}, \lambda_{1}\right)\right)-X\left(\operatorname{proj}_{\mathcal{W}_{\epsilon}}\left(x_{2}, \lambda_{2}\right)\right)\right\| \\
& \leq K\left\|\operatorname{proj}_{\mathcal{W}_{\epsilon}}\left(x_{1}, \lambda_{1}\right)-\operatorname{proj}_{\mathcal{W}_{\epsilon}}\left(x_{2}, \lambda_{2}\right)\right\| \\
& \leq K\left\|\left(x_{1}, \lambda_{1}\right)-\left(x_{2}, \lambda_{2}\right)\right\| .
\end{aligned}
$$

The last inequality follows from the Lipschitz property of the map proj $j_{\mathcal{W}_{\epsilon}}$ (cf. Section 2.3).

Next, we employ Proposition 2.2 to establish the existence, uniqueness, and continuity with respect to the initial condition of the solutions of the projected dynamical system, $X_{\mathrm{p}-\mathrm{d}}^{\mathcal{W}_{\epsilon}}$, associated with $X^{\mathcal{W}_{\epsilon}}$ and $\mathbb{R}^{n} \times \mathbb{R}_{\geq 0}^{m}$. Our proof then concludes by showing that in fact all solutions of the projected system $X_{\mathrm{p} \text {-d }}^{\mathcal{W}_{\epsilon}}$ starting in $\mathcal{W}_{\epsilon} \cap \mathbb{R}^{n} \times \mathbb{R}_{\geq 0}^{m}$ are in one-to-one correspondence with the solutions of $X_{\mathrm{p}-\mathrm{d}}$ starting in $\mathcal{W}_{\epsilon} \cap \mathbb{R}^{n} \times \mathbb{R}_{\geq 0}^{m}$. Let $X_{\mathrm{p}-\mathrm{d}}^{\mathcal{W}_{\epsilon}}: \mathbb{R}^{n} \times \mathbb{R}_{\geq 0}^{m} \rightarrow \mathbb{R}^{n} \times \mathbb{R}^{m}$ be the map obtained by projecting $X^{\mathcal{W}_{\epsilon}}$ with respect to $\mathbb{R}^{n} \times \mathbb{R}_{\geq 0}^{m}$,

$$
X_{\mathrm{p}-\mathrm{d}}^{\mathcal{W}_{\epsilon}}(x, \lambda)=\Pi_{\mathbb{R}^{n} \times \mathbb{R}_{\geq 0}^{m}}\left((x, \lambda), X^{\mathcal{W}_{\epsilon}}(x, \lambda)\right),
$$

for all $(x, \lambda) \in \mathbb{R}^{n} \times \mathbb{R}_{\geq 0}^{m}$. Since $X_{\mathrm{p}-\mathrm{d}}$ is the projection of $X$ with respect to 
$\mathbb{R}^{n} \times \mathbb{R}_{\geq 0}^{m}$, we deduce that $X_{\mathrm{p}-\mathrm{d}}^{\mathcal{W}_{\epsilon}}=X_{\mathrm{p}-\mathrm{d}}$ over the set $\mathcal{W}_{\epsilon} \cap \mathbb{R}^{n} \times \mathbb{R}_{\geq 0}^{m}$. Since $X^{\mathcal{W}_{\epsilon}}$ is Lipschitz, following Proposition 2.2, we obtain that starting from any point in $\mathbb{R}^{n} \times \mathbb{R}_{\geq 0}^{m}$, a unique solution of $X_{\mathrm{p}-\mathrm{d}}^{\mathcal{W}_{\epsilon}}$ exists over $[0, \infty)$ and is continuous with respect to the initial condition. Consider any solution $t \mapsto(\tilde{x}(t), \tilde{\lambda}(t))$ of $X_{\mathrm{p}-\mathrm{d}}^{\mathcal{W}_{\epsilon}}$ that starts in $\mathcal{W}_{\epsilon} \cap \mathbb{R}^{n} \times \mathbb{R}_{\geq 0}^{m}$. Note that since the solution is absolutely continuous and $V$ is continuously differentiable, the map $t \mapsto V(\tilde{x}(t), \tilde{\lambda}(t))$ is differentiable almost everywhere on $[0, \infty)$, and hence

$$
\frac{d}{d t} V(\tilde{x}(t), \tilde{\lambda}(t))=\mathcal{L}_{X_{\mathrm{p}-d}^{\mathcal{W}_{\epsilon}} V} V(\tilde{x}(t), \tilde{\lambda}(t))
$$

almost everywhere on $[0, \infty)$. From Lemma 4.1 and the fact that $\mathcal{L}_{X_{\mathrm{p}-d}^{\mathcal{W}_{\epsilon}} V}$ and $\mathcal{L}_{X_{\mathrm{p}-\mathrm{d}}} V$ are the same over $\mathcal{W}_{\epsilon} \cap \mathbb{R}^{n} \times \mathbb{R}_{\geq 0}^{m}$, we conclude that $V$ is non-increasing along the solution. This means the solution remains in the set $\mathcal{W}_{\epsilon} \cap \mathbb{R}^{n} \times \mathbb{R}_{\geq 0}^{m}$. Finally, since $X_{\mathrm{p}-\mathrm{d}}^{\mathcal{\mathcal { W } _ { \epsilon }}}$ and $X_{\mathrm{p}-\mathrm{d}}$ are same on $\mathcal{W}_{\epsilon} \cap \mathbb{R}^{n} \times \mathbb{R}_{\geq 0}^{m}$, we conclude that $t \mapsto(\tilde{x}(t), \tilde{\lambda}(t))$ is also a solution of $X_{\mathrm{p}-\mathrm{d}}$. Therefore, starting at any point in $\mathcal{W}_{\epsilon} \cap \mathbb{R}^{n} \times \mathbb{R}_{\geq 0}^{m}$, a solution of $X_{\mathrm{p}-\mathrm{d}}$ exists. Using Lemma 4.1, one can show that, if a solution of $X_{\mathrm{p}-\mathrm{d}}$ that starts from a point in $\mathcal{W}_{\epsilon} \cap \mathbb{R}^{n} \times \mathbb{R}_{\geq 0}^{m}$ exists, then it remains in $\mathcal{W}_{\epsilon} \cap \mathbb{R}^{n} \times \mathbb{R}_{\geq 0}^{m}$ and so is a solution of $X_{\mathrm{p}-\mathrm{d}}^{\mathcal{\mathcal { W } _ { \epsilon }}}$. This, combined with the uniqueness of solutions of $X_{\mathrm{p}-\mathrm{d}}^{\mathcal{W}}$, implies that a unique solution of $X_{\mathrm{p}-\mathrm{d}}$ exists starting from any point in $\mathcal{W}_{\epsilon} \cap \mathbb{R}^{n} \times \mathbb{R}_{\geq 0}^{m}$. In particular, this is true for the point $(x(0), \lambda(0))$. Finally, from the continuity of solutions of $X_{\mathrm{p}-\mathrm{d}}^{\mathcal{W}_{\epsilon}}$ and the one-to-one correspondence of solutions of $X_{\mathrm{p}-\mathrm{d}}$ and $X_{\mathrm{p}-\mathrm{d}}^{\mathcal{W}_{\epsilon}}$ starting $\mathcal{W}_{\epsilon} \cap \mathbb{R}^{n} \times \mathbb{R}_{\geq 0}^{m}$, we conclude the continuity with respect to initial condition for solutions of $X_{\mathrm{p}-\mathrm{d}}$ starting in $V^{-1}(x(0), \lambda(0))$. Since $(x(0), \lambda(0))$ is arbitrary, the result follows.

The next result states the invariance of the omega-limit set of any solution of the primal-dual dynamics. This ensures that all hypotheses of the invariance principle for Caratheodory solutions of discontinuous dynamical systems, cf. Proposition 2.1, are satisfied.

Lemma 4.4. (Omega-limit set of solution of primal-dual dynamics is invariant): The omega-limit set of any solution of the primal-dual dynamics starting from any point in $\mathbb{R}^{n} \times \mathbb{R}_{\geq 0}^{m}$ is invariant under (6).

The proof of Lemma 4.4 follows the same line of argumentation that the proof of invariance of omega-limit sets of solutions of locally Lipschitz vector 
fields, cf. [10, Lemma 4.1]. We are now ready to establish our main result, the asymptotic convergence of the solutions of the primal-dual dynamics to a solution of the constrained optimization problem.

Theorem 4.5. (Convergence of the primal-dual dynamics to a primal-dual optimizer): The set of primal-dual solutions of (3) is globally asymptotically stable on $\mathbb{R}^{n} \times \mathbb{R}_{\geq 0}^{m}$ under the primal-dual dynamics (6), and the convergence of each solution is to a point.

Proof. Let $\left(x_{*}, \lambda_{*}\right) \in \mathbf{X} \times \Lambda$ and consider the function $V$ defined in (8). For $\delta>0$, consider the compact set $\mathcal{S}=V^{-1}(\leq \delta) \cap\left(\mathbb{R}^{n} \times \mathbb{R}_{\geq 0}^{m}\right)$. From Lemma 4.3, we deduce that a unique solution of $X_{\mathrm{p}-\mathrm{d}}$ exists starting from any point in $\mathcal{S}$, which remains in $\mathcal{S}$. Moreover, from Lemma 4.4, the omega-limit set of each solution starting from any point in $\mathcal{S}$ is invariant. Finally, from Lemma 4.1, $\mathcal{L}_{X_{\mathrm{p}-\mathrm{d}}} V(x, \lambda) \leq 0$ for all $(x, \lambda) \in \mathcal{S}$. Therefore, Proposition 2.1 implies that any solution of $X_{\mathrm{p}-\mathrm{d}}$ staring in $\mathcal{S}$ converges to the largest invariant set $M$ contained in $\operatorname{cl}(Z)$, where $Z=\left\{(x, \lambda) \in \mathcal{S} \mid \mathcal{L}_{X_{\mathrm{p}-\mathrm{d}}} V(x, \lambda)=0\right\}$. From the proof of Lemma $4.1, \mathcal{L}_{X_{\mathrm{p}-\mathrm{d}}} V(x, \lambda)=0$ implies

$$
\begin{aligned}
L\left(x_{*}, \lambda_{*}\right)-L\left(x_{*}, \lambda\right) & =0, \\
L\left(x, \lambda_{*}\right)-L\left(x_{*}, \lambda_{*}\right) & =0, \\
\left(\lambda_{i}-\left(\lambda_{*}\right)_{i}\right)\left(\left[g_{i}(x)\right]_{\lambda_{i}}^{+}-g_{i}(x)\right) & =0, \quad \text { for all } i \in\{1, \ldots, m\} .
\end{aligned}
$$

Since $f$ is strictly concave, so is the function $x \mapsto L\left(x, \lambda_{*}\right)$ and thus $L\left(x, \lambda_{*}\right)=$ $L\left(x_{*}, \lambda_{*}\right)$ implies $x=x_{*}$. The equality $L\left(x_{*}, \lambda_{*}\right)-L\left(x_{*}, \lambda\right)=0$ implies $\lambda^{\top} g\left(x_{*}\right)=0$. Therefore $Z=\left\{(x, \lambda) \in \mathcal{S} \mid x=x_{*}, \lambda^{\top} g\left(x_{*}\right)=0\right\}$ is closed. Let $\left(x_{*}, \lambda\right) \in M \subset Z$. The solution of $X_{\mathrm{p}-\mathrm{d}}$ starting at $\left(x_{*}, \lambda\right)$ remains in $M$ (and hence in $Z$ ) only if $\nabla f\left(x_{*}\right)-\sum_{i=1}^{m} \lambda_{i} \nabla g_{i}\left(x_{*}\right)=0$. This implies that $\left(x_{*}, \lambda\right)$ satisfies the KKT conditions (5) and hence, $M \subset \mathrm{X} \times \Lambda$. Since the initial choice $\delta>0$ is arbitrary, we conclude that the set $X \times \Lambda$ is globally asymptotically stable on $\mathbb{R}^{n} \times \mathbb{R}_{\geq 0}^{m}$. Finally, we note that convergence is to a point in $\mathrm{X} \times \Lambda$. This is equivalent to saying that the omega-limit set $\Omega(x, \lambda) \subset \mathrm{X} \times \Lambda$ of any solution $t \mapsto(x(t), \lambda(t))$ of $X_{\mathrm{p}-\mathrm{d}}$ is a singleton. This fact follows from the definition of omega-limit set and the fact that, by Lemma 4.1, primal-dual optimizers are Lyapunov stable. This concludes the proof.

Remark 4.6. (Alternative proof strategy via evolution variational inequal- 
ities): We briefly describe here an alternative proof strategy to the one we have used here to establish the asymptotic convergence of the primal-dual dynamics. The Caratheodory solutions of the primal-dual dynamics can also be seen as solutions of an evolution variational inequality (EVI) problem [18]. Then, one can show that the resulting EVI problem has a unique solution starting from each point in $\mathbb{R}^{n} \times \mathbb{R}_{\geq 0}^{m}$, which moreover remains in $\mathbb{R}^{n} \times \mathbb{R}_{\geq 0}^{m}$. With this in place, the LaSalle Invariance Principle [18, Theorem 4] for the solutions of the EVI problem can be applied to conclude the convergence to the set of primal-dual optimizers.

Remark 4.7. (Primal-dual dynamics with gains): In power network optimization problems $[7,8,9]$ and network congestion control problems [19, 20], it is common to see generalizations of the primal-dual dynamics involving gain matrices. Formally, these dynamics take the form

$$
\begin{aligned}
& \dot{x}=K_{1} \nabla_{x} L(x, \lambda), \\
& \dot{\lambda}=K_{2}\left[-\nabla_{\lambda} L(x, \lambda)\right]_{\lambda}^{+},
\end{aligned}
$$

where $K_{1} \in \mathbb{R}^{n \times n}$ and $K_{2} \in \mathbb{R}^{m \times m}$ are diagonal, positive definite matrices. In such cases, the analysis performed here can be replicated following the same steps but using instead the Lyapunov function

$$
V^{\prime}(x, \lambda)=\frac{1}{2}\left(\left(x-x_{*}\right)^{\top} K_{1}^{-1}\left(x-x_{*}\right)+\left(\lambda-\lambda_{*}\right)^{\top} K_{2}^{-1}\left(\lambda-\lambda_{*}\right)\right),
$$

to establish the required monotonicity and convergence properties of (10).

\section{Conclusions}

We have considered the primal-dual dynamics for a constrained concave optimization problem and established the asymptotic convergence of its Caratheodory solutions to a primal-dual optimizer using classical notions from stability theory. Our technical approach has employed results from projected dynamical systems to establish existence, uniqueness, and continuity of the solutions, and the invariance principle for discontinuous Caratheodory systems to characterize their asymptotic convergence. We have also shown by means of a counterexample how a proof strategy based on interpreting the primal-dual dynamics as a hybrid automaton is not valid in general because 
of the lack of continuity (understood in the hybrid sense) of the solutions. The technical approach presented in the paper opens up the possibility of rigorously characterizing the robustness properties of the primal-dual dynamics against unmodeled dynamics, disturbances, and noise. Motivated by applications to power networks, we also plan to explore the design of discontinuous dynamics that can find the solutions to semidefinite programs and quadratically constrained quadratic programs.

\section{Acknowledgements}

The first and the third author wish to thank Dr. Bahman Gharesifard and Dr. Dean Richert for fruitful discussions on the primal-dual dynamics. This

research was partially supported by NSF Award ECCS-1307176, Los Alamos National Lab through a DoE grant, and DTRA under grant 11376437.

\section{References}

[1] K. Arrow, L. Hurwitz, H. Uzawa, Studies in Linear and Non-Linear Programming, Stanford University Press, Stanford, California, 1958.

[2] T. Kose, Solutions of saddle value problems by differential equations, Econometrica 24 (1) (1956) 59-70.

[3] D. Feijer, F. Paganini, Stability of primal-dual gradient dynamics and applications to network optimization, Automatica 46 (2010) 1974-1981.

[4] J. Chen, V. K. N. Lau, Convergence analysis of saddle point problems in time varying wireless systems - control theoretical approach, IEEE Transactions on Signal Processing 60 (1) (2012) 443-452.

[5] A. Ferragut, F. Paganini, Network resource allocation for users with multiple connections: fairness and stability, IEEE/ACM Transactions on Networking 22 (2) (2014) 349-362.

[6] X. Ma, N. Elia, A distributed continuous-time gradient dynamics approach for the active power loss minimizations, in: Allerton Conf. on Communications, Control and Computing, Monticello, IL, 2013, pp. 100-106.

[7] C. Zhao, U. Topcu, N. Li, S. Low, Design and stability of load-side primary frequency control in power systems, IEEE Transactions on Automatic Control 59 (5) (2014) 1177-1189. 
[8] E. Mallada, C. Zhao, S. Low, Optimal load-side control for frequency regulation in smart grids, in: Allerton Conf. on Communications, Control and Computing, Monticello, IL, 2014, pp. 731-738.

[9] X. Zhang, A. Papachristodoulou, Distributed dynamic feedback control for smart power networks with tree topology, in: American Control Conference, Portland, OR, 2014, pp. 1156-1161.

[10] H. K. Khalil, Nonlinear Systems, 3rd Edition, Prentice Hall, 2002.

[11] J. Lygeros, K. H. Johansson, S. N. Simić, J. Zhang, S. S. Sastry, Dynamical properties of hybrid automata, IEEE Transactions on Automatic Control 48 (1) (2003) 2-17.

[12] A. Nagurney, D. Zhang, Projected Dynamical Systems and Variational Inequalities with Applications, Vol. 2 of International Series in Operations Research and Management Science, Kluwer Academic Publishers, Dordrecht, The Netherlands, 1996.

[13] A. Bacciotti, F. Ceragioli, Nonpathological Lyapunov functions and discontinuous Caratheodory systems, Automatica 42 (3) (2006) 453-458.

[14] J. Cortés, Discontinuous dynamical systems - a tutorial on solutions, nonsmooth analysis, and stability, IEEE Control Systems Magazine 28 (3) (2008) $36-73$.

[15] W. Rudin, Principles of Mathematical Analysis, McGraw-Hill, 1953.

[16] F. H. Clarke, Optimization and Nonsmooth Analysis, Canadian Mathematical Society Series of Monographs and Advanced Texts, Wiley, 1983.

[17] S. Boyd, L. Vandenberghe, Convex Optimization, Cambridge University Press, 2009.

[18] B. Brogliato, D. Goeleven, The Krakovskii-LaSalle invariance principle for a class of unilateral dynamical systems, Mathematics of Control, Signals and Systems 17 (1) (2005) 57-76.

[19] J. T. Wen, M. Arcak, A unifying passivity framework for network flow control, IEEE Transactions on Automatic Control 49 (2) (2004) 162-174.

[20] S. H. Low, F. Paganini, J. C. Doyle, Internet congestion control, IEEE Control Systems Magazine 22 (1) (2002) 28-43. 